\title{
Low-cost Surrogate Models for Microwave Filters
}

\author{
Natalia Leszczynska, Ivo Couckuyt and Tom Dhaene Senior Member, IEEE, Michal Mrozowski, Fellow, IEEE
}

\begin{abstract}
A novel low-cost kriging-based multivariable parametric macromodeling technique for microwave filters is presented. Kriging is used to model both the residues and poles of a microwave filter's reflection coefficient, and the zeros of the transmission coefficient. The proposed residue-pole-zero (RPZ) technique is demonstrated to efficiently model a high dimensional (8D) microwave filter with pseudoelliptic characteristics.
\end{abstract}

Index Terms-Parametric macromodeling, kriging, rational approximation, microwave filters.

\section{INTRODUCTION}

D ESIGN of microwave filters based on the full-wave electromagnetic solvers often requires a large number of computationally intensive and time-consuming simulations. To expedite the design process surrogate models whose response approximates the behaviour of a complex system can be used in lieu of electromagnetic analysis. This approach is especially attractive for design-by-optimization. However, surrogate based optimization (SBO) makes sense only if the model is sufficiently accurate and cheap to build. Unfortunately, when the number of design variables increases, the cost of model construction often increases exponentially. For this reason it is essential to investigate techniques that are capable of evaluating the coefficients on multivariate models with as few electromagnetic simulations as possible.

A good problem formulation is a crucial aspect often overlooked in surrogate modeling. Often the values of the device's response at selected frequency points are considered [1] [3]. For microwave circuits this implies creating a surrogate model of scattering parameters. This obvious choice does not have to be a good one. In [2], [3], so-called response feature points are utilized for modeling a scattering response. This method has been shown to give good results in the modeling of the amplitude of the transmission coefficient, provided the consistency of the feature points is maintained across a training set. Recently, several approaches based on the parametrization of rational models have been presented [4], [5]. In [4], interpolation of so-called root macromodels with sequential sampling is presented. In [5], neural networks are trained to learn the relationship between residues and poles of the rational model approximating scattering matrix and the geometrical parameters. In this approach, relatively many fullwave response samples were needed to build a precise sur-

N. Leszczynska and M. Mrozowski are with Department of Electronics, Telecommunication and Informatics, WiComm Center of Excellence, The Gdańsk University of Technology, Gdańsk, Poland, e-mails: natalia.leszczynska@eti.pg.gda.pl,m.mrozowski@ieee.org

I. Couckuyt and T. Dhaene are with Ghent University-iMinds, Gaston Crommenlaan 8, Bus 201,9050 Gent, Belgium.

Manuscript received February 23, 2016; revised May 2, 2016; accepted August 25, 2016. This work was supported by the Polish National Science Centre under contract UMO-2012/07/B/ST7/01241.

Ivo Couckuyt is a post-doctoral research fellow of FWO-Vlaanderen. rogate model. Moreover, this technique was demonstrated for models involving a narrow variation of geometrical parameters and, hence, the resulting model is only accurate for a specified frequency interval and cannot be used for a new design with a different center frequency and bandwidth.

In this paper, we present a new technique for low-cost surrogate modeling and design of microwave filters. Instead of using interpolated root macromodels or artificial networks advocated in [4], [5], a hierarchical approach based on vector fitting and a kriging interpolant [6] is employed to find the relationship between the residues and poles of filter's reflection coefficient as well as transmission zeros, all extracted from the full-wave frequency response, and geometrical parameters. This choice of the quantities to be modeled (RPZ) and the interpolation technique (kriging) yields high quality broadband surrogate models that are valid over a wide range of filter dimensions, and yet cheap to build. This is the main contribution of this paper. The robustness and effectiveness of the proposed RPZ kriging methodology is demonstrated by modeling a microwave filter with pseudoelliptic characteristics. The cost of setting up the RPZ kriging model involving as many as eight parameters is just 30 full-wave simulations. The quality of the high-dimensional RPZ kriging model is verified for six designs of pseudoelliptic filters with different center frequency and bandwidths.

\section{THEORY}

Our model is the rational representation of the filter characteristics, as a function of the geometrical parameters. The reflection coefficient is represented in the residue-pole form (1), while its transmission coefficient is presented using the zero-pole (2) formulation, where $s=j \omega$. Both, $S_{11}$ and $S_{21}$ have common poles $\left(p_{i}\right)$.

$$
S_{11}(s)=\sum_{i=1}^{N} \frac{r_{i}}{s-p_{i}}, \quad(1) \quad S_{21}(s)=\frac{\prod_{i=1}^{N_{z}}\left(s-z_{i}\right)}{\prod_{i=1}^{N}\left(s-p_{i}\right)} .
$$

Using a different representation for $S_{11}$ (residue-pole) and $S_{21}$ (zero-pole) is essential for obtaining compact models. This can be explained as follows. The reflection response consists of $N$ complex conjugate pairs of residues $\left(r_{i}\right)$ and $N$ complex conjugate pairs of poles $\left(p_{i}\right)$, where $N$ is the filter order. However, the number of transmission zeros $\left(z_{i}\right)$ in the circuit is equal to $N_{z}$, which in most practical cases is lower than $N$. As a result, the RPZ surrogate model of an $N$ th order filter with $N_{z}$ transmission zeros has $4 N$ plus $2 N_{z}$ scalable variables compared to $6 N$ scalable parameters needed when the RP representation is used for both $S_{11}$ and $S_{21}$ (real and imaginary parts of residues, poles and zeros are modelled separately). In fact, $4 N$ plus $2 N_{z}$ independent surrogate models are created.

The computations of all data needed for model construction proceed as follows. First, the residues, poles and zeros for each 
sample are identified using an efficient Vector Fitting technique (VF) [7] based on the scattering parameters computed with a full-wave simulator. At the beginning of VF interpolation, the function order is set to $2 N$ (VF yields a conjugate pair of each residue, pole or zero). To achieve good accuracy of the interpolation, the VF algorithm is invoked adaptively and the order of the function is increased as the interpolation becomes more accurate. As a result the final order of the VF model could be higher than needed for modeling the filter. The rational model of the reflection response in the vicinity of the passband is of order $N$. So, when VF stops, all insignificant poles (and corresponding residues) can be removed. The same holds for the transmission zeros. Firstly, all residues, zeros and poles with negative imaginary parts are discarded. Next, from the remaining values, the relevant residues, zeros and poles extracted from the simulated response are identified by matching them to the corresponding residues, poles and zeros of the ideal transfer function obtained from circuit synthesis [8]. After that process, the number of poles and the corresponding residues is equal to $N$, while the number of transmission zeros is $N_{z}$. Next, the kriging interpolation is used to create the surrogate model over the geometrical design parameters. For each training sample $N$ residues, $N$ poles and $N_{z}$ zeros are modeled.

Kriging is a well-known interpolation technique [9], [10]. Assume a set of $n$ samples, $X=\left\{\mathbf{x}_{1}, \ldots, \mathbf{x}_{n}\right\}^{T}$ in $d$ dimensions and $\mathbf{y}$ the associated function values, where $(\cdot)^{T}$ is the transpose of a vector or matrix.

The aim is to construct an interpolant for the given samples $\mathrm{X}$ and associated function values. In this work the kriging interpolant with a constant mean function $\alpha$ is used,

$$
\hat{y}\left(\mathbf{x}^{*}\right)=\alpha+r\left(\mathbf{x}^{*}\right) \cdot \Psi^{-1} \cdot(\mathbf{y}-\alpha \mathbf{1}),
$$

where 1 is a column vector of ones. The coefficients $\alpha$ are determined using generalized least squares.

$r\left(\mathbf{x}^{*}\right)=\left(\psi\left(\mathbf{x}^{*}, \mathbf{x}_{1}\right), \ldots, \psi\left(\mathbf{x}^{*}, \mathbf{x}_{n}\right)\right)$ is an $1 \times n$ vector of correlations between the test point $\mathrm{x}^{*}$ and the samples $X$. The $n \times n$ correlation matrix $\Psi$ is,

$$
\Psi=\left(\begin{array}{ccc}
\psi\left(\mathbf{x}_{1}, \mathbf{x}_{1}\right) & \ldots & \psi\left(\mathbf{x}_{1}, \mathbf{x}_{n}\right) \\
\vdots & \ddots & \vdots \\
\psi\left(\mathbf{x}_{n}, \mathbf{x}_{1}\right) & \ldots & \psi\left(\mathbf{x}_{n}, \mathbf{x}_{n}\right)
\end{array}\right)
$$

where $\psi(\cdot, \cdot)$ is the correlation function. $\psi(\cdot, \cdot)$ is parametrized by a set of hyperparameters $\theta$, which are identified by Maximum Likelihood Estimation (MLE). Specifically, the (negative) concentrated ln-likelihood is minimized to find the optimal hyperparameters $\theta$,

$$
-\ln \left(\mathcal{L}_{\text {marginal }}\right)=-\frac{n}{2} \ln \sigma^{2}-\frac{1}{2} \ln (|\Psi|)
$$

where the signal variance $\sigma^{2}=\frac{1}{n}(\mathbf{y}-\mathbf{1} \alpha)^{T} \Psi^{-1}(\mathbf{y}-\mathbf{1} \alpha)$. |.| denotes the determinant of a matrix. The computational complexity is governed by the inverse of the correlation matrix $\Psi$ which is $O\left(n^{3}\right)$ in the number of samples using Cholesky decomposition.

The popular Gaussian correlation functions assumes the underlying response surface to be infinite differentiable, which

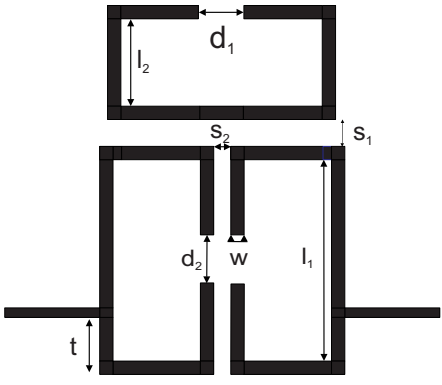

Fig. 1: Geometry of an open-loop bandpass filter

is unrealistical for many engineering problems. Hence, in this work we adopt the Matérn correlation function with $\nu=5 / 2$ as it is twice differentiable [11],

$$
\psi\left(\mathbf{x}, \mathbf{x}^{\prime}\right)_{\nu=5 / 2}=\left(1+\sqrt{5} l+\frac{5 l^{2}}{3}\right) \exp (-\sqrt{5} l),
$$

with $l=\sqrt{\sum_{i=1}^{d} \theta_{i}\left(x_{i}-x_{i}^{\prime}\right)^{2}}$.

\section{NUMERICAL EXAMPLE}

The example is a third-order open-loop bandpass filter in a triplet configuration [12]. The filter is parametrized using 8 geometrical parameters (Fig. 1), $\mathbf{x}=\left[\begin{array}{lllllll}l_{1} & l_{2} & d_{1} & d_{2} & s_{1} & s_{2} & t w\end{array}\right]^{T}$ (all dimensions in $\mathrm{mm}$ ). The variable interval for construction

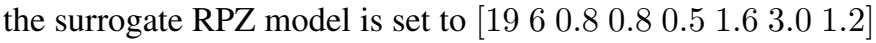
- $\left[\begin{array}{llllllll}21 & 9 & 1.25 & 1.25 & 1.0 & 2.0 & 4.2 & 1.8\end{array}\right]$. The width of feed lines set to $1 \mathrm{~mm}$, respectively. The dielectric constant is equal to 10.8 , and the substrate height is 1.27 millimetres. Three complex residues and poles $(N=3)$ of the reflection coefficient and one complex transmission zero $\left(N_{z}=1\right)$ of the microwave filter are modeled over the frequency range [0.8-1.15] GHz. The total number of independent surrogate models (scalable parameters) is 14 , since the real and imaginary parts are modeled separately. The EM response is obtained from the full-wave solver Momentum.

The kriging model is built using a research platform for surrogate modeling, the SUrrogate MOdeling (SUMO) toolbox [13]. The total budget of training samples is only 30 electromagnetic simulations in the 8D design space, arranged in a (maxi-min) Latin Hypercube Design using the translational propagation algorithm. The model accuracy is verified using 5fold cross-validation, resulting in a mean root relative squared error of 0.1661 (the coefficient of determination, $R^{2}$ is 0.97 ).

For comparison we have applied kriging to model the magnitude of reflection $S_{11}$ and transmission $S_{21}$ coefficient and to create model based on real and imaginary part of $S_{11}$ and $S_{21}$. We used the same training set. The mean root relative squared error 1.3966 and 0.6744 was obtained for the first and the second kriging model, respectively. The comparison between RPZ models and real, imaginary scattering parameters surrogate models (Sparam) is presented in Fig. 2 a-c).

To verify the accuracy of the model we design-byoptimization six different filters using the scalable RPZ kriging model and compared the surrogate-based filter response with the electromagnetic solver response evaluated at the corresponding optimal solution. Optimization was performed using 


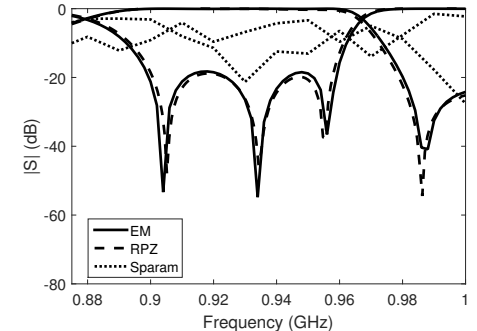

(a) $f_{0}=0.925 \mathrm{GHz}, f_{z}=0.986 \mathrm{GHz}$, $f b w=0.04$

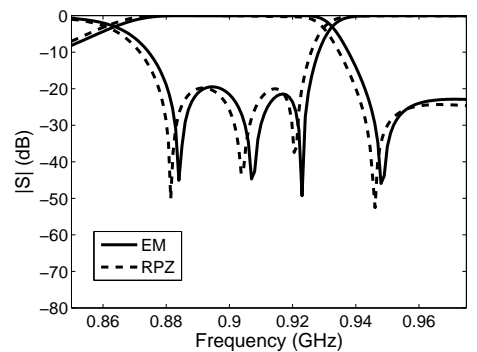

(d) $f_{0}=0.9 \mathrm{GHz}, f_{z}=0.948 \mathrm{GHz}$, fbw $=0.05$

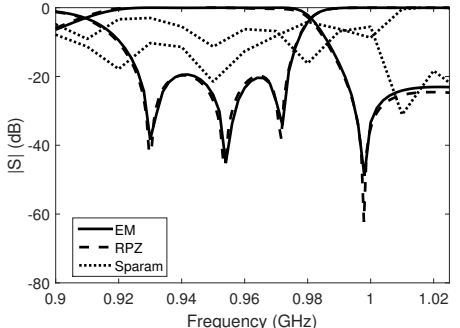

(b) $f_{0}=0.95 \mathrm{GHz}, f_{z}=0.998 \mathrm{GHz}$, fbw $=0.05$

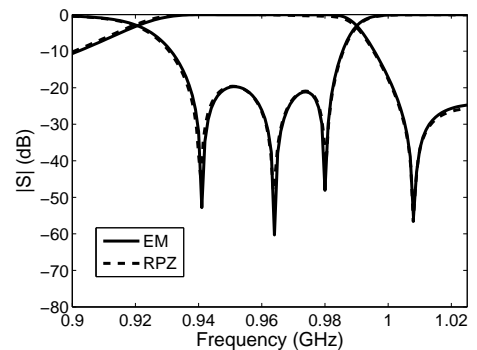

(e) $f_{0}=0.96 \mathrm{GHz}, f_{z}=1.008 \mathrm{GHz}$, fbw $=0.045$

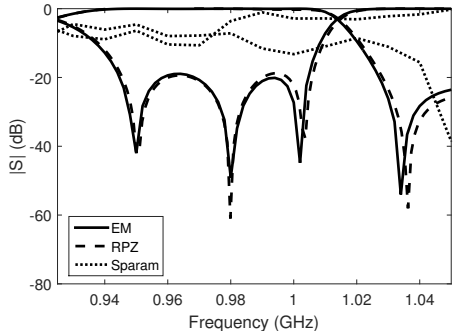

(c) $f_{0}=0.975 \mathrm{GHz}, f_{z}=1.034 \mathrm{GHz}$, fbw $=0.06$

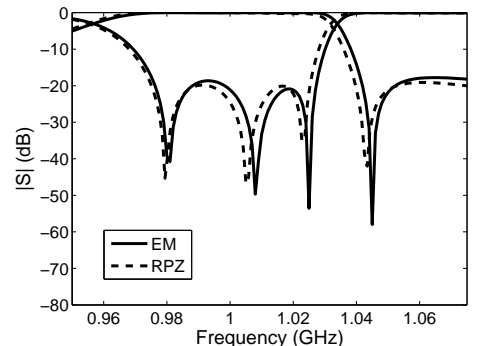

(f) $f_{0}=1.0 \mathrm{GHz}, f_{z}=1.045 \mathrm{GHz}$, fbw $=0.05$

Fig. 2: Comparison between residue-pole-zero surrogate model (RPZ) and scattering parameters surrogate models (Sparam) and corresponding full-wave (EM) model responses (upper row) and comparison between surrogate model (RPZ) optimization results and corresponding full-wave (EM) model responses (lower row).

a cost function based on the location of zeros and poles of filter's transfer and reflection function [8]. To this end, residues predicted by the constructed RPZ kriging surrogate model are converted to reflection zeros.

The designs on which the RPZ surrogate model was tested involved a variety of specifications, including the center frequency $\left(f_{0}\right)$ over the range $0.9-1.0 \mathrm{GHz}$, the fractional bandwidth $(f b w)$ in the following interval 0.04-0.06 and different position of transmission zeros $\left(f_{z}\right)$. The return loss level in all cases is set to $20 \mathrm{~dB}$. The comparison between the optimized response of the surrogate model and the full-wave characteristics are presented in Fig. 2 a-f. It is seen that in all cases the RPZ surrogate models and EM simulations responses are in good agreement.

\section{SUMMARY}

A mixed residue-pole-zero (RPZ) representation of a filter's transfer and reflection coefficient has been proposed for kriging-based modeling of a pseudoelliptic microwave bandpass filter. The RPZ model was constructed for a high dimensional design space for a wide range of geometrical parameters with using only 30 training samples. The accuracy of the presented method has been verified by design and optimization of six different bandpass filters. Despite the high number of geometric parameters (8), a very low number of computational expansive samples can be used to build surrogate models.

\section{REFERENCES}

[1] A. Lamecki, P. Kozakowski, and M. Mrozowski, "Efficient implementation of the Cauchy method for automated CAD-model construction,"
Microwave and Wireless Components Letters, IEEE, vol. 13, no. 7, pp. 268-270, 2003

[2] S. Koziel, Q. S. Cheng, and J. W. Bandler, "Feature-based surrogates for low-cost microwave modelling and optimisation," IET Microwaves, Antennas Propagation, vol. 9, no. 15, pp. 1706-1712, 2015.

[3] S. Koziel and J. W. Bandler, IEEE Transactions on Microwave Theory and Techniques.

[4] F. Ferranti, T. Dhaene, and L. Knockaert, "Compact and passive parametric macromodeling using reference macromodels and positive interpolation operators," Components, Packaging and Manufacturing Technology, IEEE Transactions on, vol. 2, no. 12, pp. 2080-2088, Dec 2012.

[5] F. Feng, C. Zhang, J. Ma, and Q.-J. Zhang, "Parametric modeling of EM behavior of microwave components using combined neural networks and pole-residue-based transfer functions," Microwave Theory and Techniques, IEEE Transactions on, vol. 64, no. 1, pp. 60-77, Jan 2016.

[6] I. Couckuyt, F. Declercq, T. Dhaene, H. Rogier, and L. Knockaert, "Surrogate-based infill optimization applied to electromagnetic problems," International Journal of RF and Microwave Computer-Aided Engineering, vol. 20, no. 5, pp. 492-501, 2010.

[7] B. Gustavsen and A. Semlyen, "Rational approximation of frequency domain responses by vector fitting," IEEE Trans. Power Del., vol. 14, no. 3, pp. $1052-1061$, Jul. 1999.

[8] P. Kozakowski and M. Mrozowski, "Automated CAD of coupled resonator filters," IEEE Microw. Wireless Compon. Lett., vol. 12, no. 12, pp. $470-472$, Dec. 2002.

[9] I. Couckuyt, T. Dhaene, and P. Demeester, "ooDACE toolbox: A flexible object-oriented kriging implementation," Journal of Machine Learning Research, no. 15, pp. 3183-3186, 2014.

[10] J. Kleijnen, Design and Analysis of Simulation Experiments. John Wiley and Sons, 2008.

[11] M. Stein, Interpolation of Spatial Data: Some Theory for Kriging. Springer-Verlag, 1999.

[12] M. L. J-S. Hong, Microstrip Filters for RF/Microwave Application. John Wiley and Sons, 2001.

[13] D. Gorissen, K. Crombecq, I. Couckuyt, P. Demeester, and T. Dhaene, "A surrogate modeling and adaptive sampling toolbox for computer based design," Journal of Machine Learning Research, no. 11, pp. 20512055,2010 